\title{
Maximum principles around an eigenvalue with constant eigenfunctions
}

\author{
J. Campos ${ }^{1}$, J. Mawhin ${ }^{2}$ and R. Ortega ${ }^{1}$ \\ ${ }^{1}$ Departamento de Matemática Aplicada, Facultad de Ciencias \\ Universidad de Granada, 18071-Granada, Spain. \\ ${ }^{2}$ Institut de Mathématique \\ Université Catholique de Louvain, B-1348 Louvain-la-Neuve, Belgium \\ campos@ugr.es, jean.mawhin@uclouvain.be, rortega@ugr.es
}

\begin{abstract}
A class of linear operators $L+\lambda I$ between suitable function spaces is considered, when 0 is an eigenvalue of $L$ with constant eigenfunctions. It is proved that $L+\lambda I$ satisfies a strong maximum principle when $\lambda$ belongs to a suitable pointed left-neighborhood of 0 , and satisfies a strong uniform antimaximum principle when $\lambda$ belongs to a suitable pointed right-neighborhood of 0 . Applications are given to various type of ordinary or partial differential operators with periodic or Neumann boundary conditions.
\end{abstract}

MSC (2000) : Primary 35B50; Secondary 34C25, 35J40, 35L20, 47B60

Key words : maximum principle, anti-maximum principle, periodic solutions, polyharmonic operators, telegraph equation

\section{Introduction}

Consider the problem

$$
\Delta u+\lambda u=f(x) \text { in } D, \quad B u=0 \text { in } \partial D,
$$

where $D$ is a smooth bounded domain in $\mathbb{R}^{N}$ and $B u=0$ represents either the Dirichlet or Neumann homogenous boundary conditions.

It is a standard consequence of the maximum principle (MP) that if $\lambda<\lambda_{1}$, where $\lambda_{1}$ represents the principal eigenvalue of $-\Delta$ under the corresponding boundary conditions, and if $f$ is a nonnegative function, then 
the solution $u$ of (1) is nonpositive in $\bar{D}$. Indeed, a stronger conclusion holds for (1), namely a strong maximum principle (SMP) : if $f$ is nonnegative and not identically zero, then $u$ is negative in $D$. We refer to the classical book [29] for more details and early references, to [4] for a general statement, and to the recent monograph [30] for more details on this important tool. Those references essentially deal with partial differential equations or systems of elliptic or of parabolic type.

In 1979, Clément and Pelletier [12] investigated the problem (1) in the situation where $\lambda>\lambda_{1}$ and proved the following anti-maximum principle (AMP) : Given a nonnegative function $f$, there exists $\delta=\delta(f)>0$ such that if $\lambda_{1}<\lambda<\lambda_{1}+\delta$, then any solution $u$ of (1) is nonnegative in $D$. Furthermore, a strong anti-maximum principle (SAMP) holds in this case : if $f$ is nonnegative and not identically zero, then $u$ is positive in $D$. They also showed in [12] that $\delta$ can be taken independent of $f$ for the Neumann problem in dimension $N=1$, in which case one speaks of a uniform antimaximum principle (UAMP). Recent work with those (possibly strong) AMP and UAMP for linear elliptic operators with various boundary conditions include the papers of Hess [21] (AMP for elliptic problems with weight), de Figueiredo-Gossez [15] (connection to the Fučik spectrum), Birindelli [5] (irregular domains), Takač [35] (MP and AMP for abstract linear elliptic boundary value problems in strongly ordered space), Cabada-Lois [10] (UAMP for higher order ordinary differential operators with periodic boundary conditions), Sweers [34] (exact $L^{p}$ space where $f$ should be taken), Pinchover [28] (MP and AMP via perturbation theory of positive solutions), Alziary-Fleckinger-Takáč [1] (MP and AMP for Schrödinger equation in $\mathbb{R}^{2}$ ), Godoy-Gossez-Paczka $[17,18]$ (AMP and UAMP for Dirichlet, Neumann or Robin problems with weight), Clément-Sweers $[13,14]$ (UAMP for second or higher order elliptic operators with homogeneous boundary conditions), Stavrakakis-de Thélin [33] (AMP for elliptic equation on $\mathbb{R}^{N}$ ), Grunau-Sweers [20] (optimal conditions for AMP or UAMP for polyharmonic boundary value problems), BartenevaCabada-Ignatyev [3] and Reichel [31] (MP and AMP for second order ordinary differential operators with variable coefficients), Arcoya-Gámez [2] and Shi Junping [32] (proofs of AMP using bifurcation) and others.

In the case of Neumann problem in dimension one

$$
u^{\prime \prime}+\lambda u=f(x) \quad \text { in } \quad(0, \pi), \quad u^{\prime}(0)=0=u^{\prime}(\pi)
$$

zero is the principal eigenvalue, and, if $f \geq 0$ on $(0, \pi)$, the SMP tells that $u<0$ on $(0, \pi)$ when $\lambda<0$, and the SUAMP tells that $u>0$ on $(0, \pi)$ when $\lambda \in(0,1 / 4]$. In other words, $\lambda u>0$ when $\lambda \in(-\infty, 0) \cup(0,1 / 4]$. 
Less standard maximum principles have been obtained recently for the time-periodic or the time-bounded solutions of telegraph equations of the form

$$
u_{t t}+c u_{t}-\Delta u+\lambda u=f(t, x)
$$

with periodic spatial boundary conditions (see $[27,23,24]$ ). In the case of time-periodic solutions, a SMP holds for $\lambda \in\left(0, \lambda_{+}\right]$, for some finite $\lambda_{+}>0$ depending upon $c$. A natural question is the existence of an anti-maximum principle for such a problem.

The aim of this paper is to identify a class of abstract linear operators $L+\lambda I$ acting on some function spaces, $\lambda=0$ being an eigenvalue of $L$ with constant eigenfunctions, for which a MP holds when $\lambda \in\left[\lambda_{-}, 0\right)$, a SMP holds when $\lambda \in\left(\lambda_{-}, 0\right)$, a UAMP holds when $\lambda \in\left(0, \lambda_{+}\right]$, and a SUAMP holds when $\lambda \in\left(0, \lambda_{+}\right)$, for some $-\infty \leq \lambda_{-}<0<\lambda_{+} \leq+\infty$. A precise statement is given in Theorem 1 of Section 2. The proof is based upon a detailed study of the resolvent operator $R_{\lambda}$ of $L$.

Various applications are given in Section 3, namely to linear differential operators of arbitrary order with constant coefficients and periodic boundary conditions, first order difference equations with periodic boundary conditions, a two-point boundary value problem of order four, polyharmonic operators with Neumann-type boundary conditions on a smooth bounded domain of $\mathbb{R}^{N}$, and some hyperbolic operators on a torus, generalizing the telegraph equation with periodic boundary conditions in space and time. This shows in particular that, in this case, a SUAMP holds in addition to the SMP proved in [27].

\section{Abstract setting of the results}

Let $\Omega$ be a compact metric space and let $\mu$ be a positive and bounded measure over $\Omega$. The notion of measure is understood as in [16]. We shall work with the Banach spaces $\mathcal{C}=C(\Omega)$ with its standard norm $\|u\|_{\infty}=$ $\max _{\omega \in \Omega}|u(\omega)|$ and $\mathcal{L}=L^{1}(\Omega, \mu)$ with its standard norm $\|f\|_{1}=\int_{\Omega}|f| d \mu$. Given $f \in \mathcal{L}$ we employ the notations

$$
\bar{f}=\frac{1}{\mu(\Omega)} \int_{\Omega} f d \mu, \quad \widetilde{f}=f-\bar{f}, \quad \widetilde{\mathcal{L}}=\{f \in \mathcal{L} \mid \bar{f}=0\}, \quad \widetilde{\mathcal{C}}=\mathcal{C} \cap \widetilde{\mathcal{L}} .
$$

Let $L: \operatorname{Dom}(L) \subset \mathcal{C} \rightarrow \mathcal{L}$ be a linear operator satisfying

$$
\operatorname{Ker}(L)=\{\text { constant functions }\}, \operatorname{Im}(L)=\widetilde{\mathcal{L}}
$$


and such that equation

$$
L u=\widetilde{f}
$$

has a unique solution $\widetilde{u} \in \widetilde{\mathcal{C}}$. Moreover assume that

$$
\|\widetilde{u}\|_{\infty} \leq K\|\widetilde{f}\|_{1}
$$

where $K$ only depends upon $L$. Thus $L$ is a closed Fredholm operator of index zero.

Definition 1 Given $\lambda \in \mathbb{R} \backslash\{0\}$, the operator $L+\lambda I$ satisfies a maximum principle if for each $f \in \mathcal{L}$, the equation

$$
L u+\lambda u=f, \quad u \in \operatorname{Dom}(L)
$$

has a unique solution and $\lambda u \geq 0$ for any $f \geq 0$. The maximum principle is said to be strong if $\lambda u(x)>0$ for any $x \in \Omega$ when $f \geq 0$ and $f(x)>0$ in a subset of $\Omega$ with positive measure.

Such a definition includes both maximum and anti-maximum principles. For example, if $L u=u^{\prime \prime}$ with the Neumann boundary conditions on $\Omega=[0,1]$, our definition corresponds to a maximum principle when $\lambda<0$ and to an anti-maximum principle when $\lambda>0$.

The main result of this paper is the following

Theorem 1 Assume that conditions (3) and (4) hold. Then there exist numbers $\lambda_{-}$and $\lambda_{+}$, with

$$
-\infty \leq \lambda_{-}<0<\lambda_{+} \leq+\infty
$$

such that $L+\lambda I$ has a maximum principle if and only if $\lambda \in\left[\lambda_{-}, 0\right) \cup\left(0, \lambda_{+}\right]$. Moreover if $\lambda \in\left(\lambda_{-}, 0\right) \cup\left(0, \lambda_{+}\right)$the maximum principle is strong.

Before giving the proof of Theorem 1, we introduce some notations, and prove three lemmas. The inverse of $L+\lambda I$, whenever it exists, is called the resolvent of $L$, and is denoted by $R_{\lambda}$, so that $R_{\lambda}: \mathcal{L} \rightarrow \mathcal{C}$. The partial resolvent for $\lambda=0$, denoted by $\widetilde{R}_{0}$, is the operator $\widetilde{R}_{0}: \widetilde{\mathcal{L}} \rightarrow \widetilde{\mathcal{C}}$, defined by

$$
\widetilde{u}=\widetilde{R}_{0} \tilde{f} \quad \Longleftrightarrow \quad L \widetilde{u}=\widetilde{f} .
$$

We know by assumption (4) that $\widetilde{R}_{0}$ is continuous. 
Lemma 1 There exist some $\Lambda_{1}>0$ such that, if $\lambda \in\left[-\Lambda_{1}, \Lambda_{1}\right] \backslash\{0\}$, then the resolvent $R_{\lambda}$ of $L$ exists. Moreover

$$
\left\|R_{\lambda} \widetilde{f}\right\|_{\infty} \leq\left(\frac{\left\|\widetilde{R}_{0}\right\|_{\widetilde{\mathcal{L}} \rightarrow \widetilde{\mathcal{C}}}}{1-\Lambda_{1}\left\|\widetilde{R}_{0}\right\|_{\widetilde{\mathcal{C}} \rightarrow \widetilde{\mathcal{C}}}}\right)\|\widetilde{f}\|_{1}
$$

if $\widetilde{f} \in \widetilde{\mathcal{L}}, 0<|\lambda| \leq \Lambda_{1}$.

Proof. Split equation (5) in the following way

$$
\left\{\begin{array}{c}
L \widetilde{u}+\lambda \widetilde{u}=\widetilde{f} \\
\lambda \bar{u}=\bar{f}
\end{array}\right.
$$

Using the partial resolvent of $L$, the first equation in (7) can be written as

$$
\widetilde{u}+\lambda \widetilde{R}_{0} \widetilde{u}=\widetilde{R}_{0} \widetilde{f}
$$

Let us observe that $\widetilde{R}_{0}$ is also a continuous map when restricted to $\widetilde{\mathcal{C}}$. If $|\lambda|\left\|\widetilde{R}_{0}\right\|_{\widetilde{\mathcal{C}} \rightarrow \widetilde{\mathcal{C}}}<1$ then $I+\lambda \widetilde{R}_{0}$ is invertible from $\widetilde{\mathcal{C}}$ to $\widetilde{\mathcal{C}}$ and $(8)$ is solved as

$$
\widetilde{u}=\left(I+\lambda \widetilde{R}_{0}\right)^{-1} \widetilde{R}_{0} \widetilde{f}
$$

To finish this lemma, we take $0<\Lambda_{1}<\frac{1}{\left\|\widetilde{R}_{0}\right\|_{\widetilde{\mathcal{C}} \rightarrow \widetilde{\mathcal{C}}}}$ and obtain from (8), for $|\lambda| \leq \Lambda_{1}$

$$
\begin{aligned}
\|\widetilde{u}\|_{\infty}-\Lambda_{1}\left\|\widetilde{R}_{0}\right\|_{\widetilde{\mathcal{C}} \rightarrow \widetilde{\mathcal{C}}}\|\widetilde{u}\|_{\infty} & \leq\|\widetilde{u}\|_{\infty}-|\lambda|\left\|\widetilde{R}_{0}\right\|_{\widetilde{\mathcal{C}} \rightarrow \widetilde{\mathcal{C}}}\|\widetilde{u}\|_{\infty} \\
& \leq\|(I+\lambda \widetilde{R}) \widetilde{u}\|_{\infty}=\left\|\widetilde{R_{0}} \tilde{f}\right\|_{\infty} \\
& \leq\left\|\widetilde{R}_{0}\right\|_{\widetilde{\mathcal{L}} \rightarrow \widetilde{\mathcal{C}}}\|\widetilde{f}\|_{1},
\end{aligned}
$$

and hence

$$
\|\widetilde{u}\|_{\infty} \leq\left(\frac{\left\|\widetilde{R}_{0}\right\|_{\widetilde{\mathcal{L}} \rightarrow \widetilde{\mathcal{C}}}}{1-\Lambda_{1}\left\|\widetilde{R}_{0}\right\|_{\widetilde{\mathcal{C}} \rightarrow \widetilde{\mathcal{C}}}}\right)\|\widetilde{f}\|_{1},
$$

which gives (6).

Lemma 2 There exists $\Lambda_{2} \in\left(0, \Lambda_{1}\right)$ such that, if $0<|\lambda| \leq \Lambda_{2}$, then $L+\lambda I$ has a strong maximum principle. 
Proof. If we take $f \geq 0$, then $\bar{f}=\frac{1}{\mu(\Omega)}\|f\|_{1}$, so that, using the second equation in (7),

$$
\lambda R_{\lambda}(\bar{f}+\widetilde{f})=\bar{f}+\lambda R_{\lambda}(\widetilde{f})=\frac{1}{\mu(\Omega)}\|f\|_{1}+\lambda R_{\lambda} \widetilde{f} \geq \frac{1}{\mu(\Omega)}\|f\|_{1}-|\lambda|\left\|R_{\lambda} \widetilde{f}\right\|_{\infty}
$$

and using (6), if $0<|\lambda| \leq \Lambda_{1}$,

$$
\lambda u=\lambda R_{\lambda}(\bar{f}+\widetilde{f}) \geq \frac{1}{\mu(\Omega)}\|f\|_{1}-|\lambda|\left(\frac{\left\|\widetilde{R}_{0}\right\|_{\widetilde{\mathcal{L}} \rightarrow \widetilde{\mathcal{C}}}}{1-\Lambda_{1}\left\|\widetilde{R}_{0}\right\|_{\tilde{\mathcal{C}} \rightarrow \widetilde{\mathcal{C}}}}\right)\|\tilde{f}\|_{1} .
$$

Since $\|\widetilde{f}\|_{1} \leq\|f\|_{1}+\|\bar{f}\|_{1}=2\|f\|_{1}$, the result follows by taking

$$
\Lambda_{2}<\min \left\{\Lambda_{1}, \frac{1-\Lambda_{1}\left\|\widetilde{R}_{0}\right\|_{\widetilde{\mathcal{C}} \rightarrow \widetilde{\mathcal{C}}}}{2 \mu(\Omega)\left\|\widetilde{R}_{0}\right\|_{\widetilde{\mathcal{L}} \rightarrow \widetilde{\mathcal{C}}}}\right\}
$$

Lemma 3 Assume that $L+\lambda_{0} I$ has a maximum principle for some $\lambda_{0}>0$. Then, for any $\lambda \in\left(0, \lambda_{0}\right), L+\lambda I$ has a strong maximum principle. Moreover $R_{\lambda}$ is defined for any $\lambda \in\left(0,2 \lambda_{0}\right)$ and for any $f \in \mathcal{L}$ the function $\lambda \rightarrow R_{\lambda} f$ is analytic.

Proof. A first observation is that the existence of a maximum principle implies the continuity of the resolvent from $\mathcal{C} \rightarrow \mathcal{C}$. Indeed, because of (5), $R_{\lambda_{0}} 1=\frac{1}{\lambda_{0}}$ and if $f \in \mathcal{C},-\|f\|_{\infty} \leq f \leq\|f\|_{\infty}$. So using the maximum principle,

$$
-\frac{\|f\|_{\infty}}{\lambda_{0}} \leq R_{\lambda_{0}} f \leq \frac{\|f\|_{\infty}}{\lambda_{0}}
$$

i.e.

$$
\left\|R_{\lambda_{0}}\right\|_{\mathcal{C} \rightarrow \mathcal{C}}=\frac{1}{\lambda_{0}} .
$$

Now, let us take $\lambda \in \mathbb{R}$ and write (5) as

$$
L u+\lambda_{0} u=\left(\lambda_{0}-\lambda\right) u+f,
$$

or, equivalently,

$$
u-\left(\lambda_{0}-\lambda\right) R_{\lambda_{0}} u=R_{\lambda_{0}} f .
$$

This equation is solvable if

$$
\left\|\left(\lambda_{0}-\lambda\right) R_{\lambda_{0}}\right\|_{\mathcal{C} \rightarrow \mathcal{C}}<1,
$$


which, using estimate (9), holds if

$$
\left|\frac{\lambda_{0}-\lambda}{\lambda_{0}}\right|<1 \quad \Longleftrightarrow \quad 0<\lambda<2 \lambda_{0}
$$

Thus equation (5) is solvable in $\left(0,2 \lambda_{0}\right)$.

If now $f \in \mathcal{L}$, then $R_{\lambda_{0}} f \in \mathcal{C}$ and

$$
R_{\lambda} f=\left[I-\left(\lambda_{0}-\lambda\right) R_{\lambda_{0}}\right]^{-1} R_{\lambda_{0}} f=\left(\sum_{n \in \mathbb{N}}\left(\lambda_{0}-\lambda\right)^{n} R_{\lambda_{0}}^{n}\right) R_{\lambda_{0}} f .
$$

This expression is analytic in $\lambda$, and nonnegative if $\lambda<\lambda_{0}$ and $f \geq 0$. This gives the maximum principle. To finish the lemma, it remains to show that the strong maximum principle holds for any $\lambda \in\left(0, \lambda_{0}\right)$. Take $f \geq 0$ with $\int_{\Omega} f d \mu>0$. Then from (10), if $0<\lambda_{2}<\lambda_{1}<\lambda_{0}$, one has $R_{\lambda_{2}} f \geq R_{\lambda_{1}} f$. Fix any $x \in \Omega$ and consider the function $\varphi(\lambda)=R_{\lambda} f(x), \quad \lambda \in\left(0, \lambda_{0}\right]$. The previous remarks imply that this function in non-negative and monotone non-increasing. From Lemma 2 we know that $\varphi(\lambda)>0$ if $\lambda \in\left(0, \Lambda_{2}\right]$. We invoke the analyticity of $\varphi$ in $\left(0, \lambda_{0}\right]$ and conclude that $\varphi(\lambda)>0$ everywhere in $\left(0, \lambda_{0}\right)$.

Proof of Theorem 1. It is enough to prove the result for $\lambda>0$. For the case of negative $\lambda$ it is enough to replace $L$ by $-L$. From Lemmas 2 and 3 we know that

$$
I_{+}=\{\lambda>0 \mid L+\lambda I \text { has a maximum principle }\}
$$

is a nonempty interval. Assuming that $\lambda_{+}=\sup I_{+}$is finite, it remains to prove that $\lambda_{+} \in I_{+}$. Take $\lambda_{n} \nearrow \lambda_{+}$, and select $n_{0}$ large enough such that $\lambda_{+}<2 \lambda_{n_{0}}$. Thus $R_{\lambda_{+}}$exists and we can apply the identity (10) with $\lambda_{0}=\lambda_{+}$to conclude that $u_{n}=R_{\lambda_{n}} f \rightarrow u=R_{\lambda_{+}} f$ uniformly in $\Omega$. If $f \geq 0$ then $u_{n} \geq 0$ and so $u \geq 0$.

A natural question after Theorem 1 is whether there exist some links between the numbers $\lambda_{ \pm}$and the spectrum of $-L$. To adjust to the usual notations we consider the resolvent set $\mathcal{R}$ of the operator $-L$, that is

$$
\mathcal{R}=\left\{\lambda \in \mathbb{C} \mid L+\lambda I \text { is one-to-one, onto and }(L+\lambda I)^{-1} \text { is continuous }\right\} .
$$

As usual in spectral theory the symbol $L$ also denotes the complex extension. Assuming that $\lambda_{+}$is finite we observe from the proof of Lemma 3 that the open disk of center $\lambda_{+}$and radius $\lambda_{+}$is contained in $\mathcal{R}$. The same applies 
to the disk centered at $\lambda_{-}$and of radius $\left|\lambda_{-}\right|$when this number is finite. In particular $\lambda_{ \pm}$belong to $\mathcal{R}$ and cannot be eigenvalues of $-L$. Define $\Sigma_{+}$as the largest positive number such that the open disk of center $\Sigma_{+}$and radius $\Sigma_{+}$is contained in $\mathcal{R}$. In an analogous way one can define $\Sigma_{-}$and it is now obvious that

$$
\Sigma_{-} \leq \lambda_{-}<0<\lambda_{+} \leq \Sigma_{+}
$$

with the conventions $\Sigma_{+}=+\infty$ if $\lambda_{+}=+\infty, \Sigma_{-}=-\infty$ if $\lambda_{-}=-\infty$. The next example shows that these estimates are sharp.

Let $\Omega$ be the metrizable space composed by two points $A$ and $B$. We consider the measure satisfying $\mu(\{A\})=\alpha$ and $\mu(\{B\})=\beta$, where $\alpha>0$ and $\beta>0$ are real parameters. In this case the spaces $\mathcal{C}$ and $\mathcal{L}$ can be identified to $\mathbb{R}^{2}$ with the corresponding norms

$$
\|(x, y)\|_{\infty}=\max \{|x|,|y|\} \text { and }\|(x, y)\|_{\alpha, \beta}=\alpha|x|+\beta|y| .
$$

The operator $L: \operatorname{Dom}(L)=\mathbb{R}^{2} \rightarrow \mathbb{R}^{2}$ is given by the matrix

$$
\left(\begin{array}{cc}
\beta & -\beta \\
-\alpha & \alpha
\end{array}\right)
$$

An easy computation shows that $\operatorname{Ker}(L)$ is spanned by the vector $(1,1)^{T}$ while $\operatorname{Im}(L)$ is the line of equation $\alpha x+\beta y=0$, which coincides with $\widetilde{\mathcal{L}}$. Also,

$$
(L+\lambda I)^{-1}=\frac{1}{\lambda(\alpha+\beta+\lambda)}\left(\begin{array}{cc}
\alpha+\lambda & \beta \\
\alpha & \beta+\lambda
\end{array}\right)
$$

for every $\lambda \in \mathcal{R}=\mathbb{C} \backslash\{0,-(\alpha+\beta)\}$.

The maximum principle holds whenever this matrix has non-negative coefficients. Hence $\lambda_{+}=\infty$ and $\lambda_{-}=-\min \{\alpha, \beta\}$. Since $\Sigma_{-}=-\left(\frac{\alpha+\beta}{2}\right)$ we obtain the expected inequality $\Sigma_{-} \leq \lambda_{-}$which is strict except in the case $\alpha=\beta$.

\section{Some examples}

\subsection{Periodic solutions of ordinary differential equations}

We discuss the maximum principle for problems of the type

$$
\left\{\begin{array}{l}
u^{(n)}+a_{n-1} u^{(n-1)}+\ldots+a_{1} u^{\prime}+\lambda u=f(t) \\
u \quad 2 \pi \text {-periodic }
\end{array}\right.
$$

where $a_{n-1}, a_{n-2}, \ldots a_{1}$ are real coefficients and $f$ is $2 \pi$-periodic. 
The space $\Omega$ is the quotient group $\mathbb{T}=\mathbb{R} / 2 \pi \mathbb{Z}$ and $\mu$ is the associated Haar measure. We normalize the measure so that $\mu(\mathbb{T})=2 \pi$. This just means that for any continuous function $\phi \in C(\mathbb{T})$,

$$
\int_{\mathbb{T}} \phi d \mu=\int_{0}^{2 \pi} \phi(s) d s
$$

The space $\mathcal{L}=L^{1}(\mathbb{T})$ is the usual space of periodic functions that are integrable over the period. The operator $L$ will be taken as

$$
L u=u^{(n)}+a_{n-1} u^{(n-1)}+\ldots+a_{1} u^{\prime}, \quad u \in \operatorname{Dom}(L)=W^{n, 1}(\mathbb{T}),
$$

where $W^{n, 1}(\mathbb{T})$ is the Sobolev space composed by $2 \pi$-periodic functions which are of class $C^{n-1}$ and such that $u^{(n-1)}$ is absolutely continuous. To guarantee that $\operatorname{Ker}(L)$ only contains constant functions we must impose a condition on the characteristic polynomial

$$
p(\lambda)=\lambda^{n}+a_{n-1} \lambda^{n-1}+\cdots+a_{1} \lambda,
$$

namely,

$$
p(k i) \neq 0 \text { for each } k \in \mathbb{Z} \backslash\{0\} .
$$

To prove that

$$
\operatorname{Im}(L)=\left\{f \in \mathcal{L} \mid \int_{0}^{2 \pi} f(t) d t=0\right\}
$$

we employ the Fredholm alternative. According to this principle the space $\operatorname{Im}(L)$ is composed by those functions $f$ which are orthogonal (in the $L^{2}$ sense) to the $2 \pi$-periodic solutions of $L^{\star} u=0$, where $L^{\star}$ is the adjoint operator

$$
L^{\star}(v):=(-1)^{n} v^{(n)}+(-1)^{n-1} a_{n-1} v^{(n-1)}+\ldots+(-1) a_{1} v^{\prime} .
$$

The only $2 \pi$-periodic solutions of the adjoint equation are again the constants. Indeed, the characteristic polynomial associated to $L^{\star}$ is $p^{\star}(\lambda)=$ $p(-\lambda)$ and so condition (12) holds for $p^{\star}$ and $p$ simultaneously. When $\widetilde{W}^{n, 1}(\mathbb{T})=\left\{u \in W^{n, 1}(\mathbb{T}): \bar{u}=0\right\}$ and $\widetilde{L}^{1}(\mathbb{T})=\left\{f \in L^{1}(\mathbb{T}): \bar{f}=0\right\}$ are endowed with their natural norms, so that they become Banach spaces, the map

$$
\widetilde{u} \in \widetilde{W}^{n, 1}(\mathbb{T}) \mapsto \widetilde{u}^{(n)}+a_{n-1} \widetilde{u}^{(n-1)}+\cdots+a_{1} \widetilde{u}^{\prime}=\widetilde{f} \in \widetilde{L}^{1}(\mathbb{T})
$$

is a continuous isomorphism. Therefore the inverse is also continuous and so the estimate $\|\widetilde{u}\|_{W^{n, 1}} \leq K_{1}\|\widetilde{f}\|_{1}$ holds. We are in dimension one and so 
the space $\widetilde{W}^{n, 1}(\mathbb{T})$ is immersed in $\widetilde{C}(\mathbb{T})=\{u \in C(\mathbb{T}): \bar{u}=0\}$, implying that $\|\widetilde{u}\|_{\infty} \leq K_{2}\|\widetilde{u}\|_{W^{n, 1}}$. These two estimates lead to the $L^{1}-L^{\infty}$ condition required for the applicability of Theorem 1 and we obtain maximum principles for $\lambda$ positive or negative.

For $n=1$ or 2, condition (12) always holds and the numbers $\lambda_{+}$and $\lambda_{-}$can be computed in several ways. They are $\lambda_{ \pm}= \pm \infty$ if $n=1$ and

$\lambda_{+}=\frac{1+a_{1}^{2}}{4}, \lambda_{-}=-\infty$ if $n=2$. Since every constant is explicit in this case, we can discuss the relationships with the spectrum of $-L$ and the numbers $\Sigma_{ \pm}$. For $n=1$ this spectrum is the sequence $\lambda_{k}=i k$ with $k \in \mathbb{Z}$. It lies on the imaginary axis and so $\Sigma_{ \pm}= \pm \infty$. For $n=2$ the spectrum becomes $\lambda_{k}=k^{2}-i a_{1} k, k \in \mathbb{Z}$. A computation shows that $\Sigma_{-}=-\infty$, $\Sigma_{+}=\frac{1+a_{1}^{2}}{2}=2 \lambda_{+}$.

The determination of the exact value of $\lambda_{ \pm}$is more delicate for $n \geq 3$ and we refer to $[7,8,9,10,25,26]$. See also [11] for Neumann boundary conditions and $[3,31]$ for second order operators with variable coefficients.

\subsection{Periodic solutions of difference equations}

Let $\Delta u_{m}:=u_{m+1}-u_{m}$ denote the forward difference operator, and, given the positive integer $n$, let us consider the difference equation with periodic boundary conditions

$$
\Delta u_{m}+\lambda u_{m}=f_{m} \quad(0 \leq m \leq n-1), \quad u_{0}=u_{n},
$$

where $\lambda \in \mathbb{R}$ and $f_{m} \in \mathbb{R}(0 \leq m \leq n-1)$. Problem (13) is equivalent to the problem

$$
\Delta u_{m}+\lambda u_{m}=f_{m} \quad(0 \leq m \leq n-2), \quad u_{0}-u_{n-1}+\lambda u_{m-1}=f_{n-1} .
$$

If $\Omega$ is the metrizable space composed of $n$ points $A_{0}, A_{2}, \ldots A_{n-1}$, and $\mu$ the measure on $\Omega$ satisfying $\mu\left(\left\{A_{m}\right\}\right)=1(0 \leq m \leq n-1)$, then $\mathcal{C}$ can be identified with $\mathbb{R}^{n}=\left\{u=\left(u_{0}, \ldots, u_{n-1}\right)\right\}$ endowed with the norm $\|u\|_{\infty}=$ $\max _{0 \leq m \leq n-1}\left|u_{m}\right|$, and $\mathcal{L}$ can be identified with $\mathbb{R}^{n}$ endowed with the norm $\|u\|_{1}=\sum_{m=0}^{n-1}\left|u_{m}\right|$. It is easy to see that the operator $L: \operatorname{Dom}(L)=\mathbb{R}^{n} \rightarrow$ $\mathbb{R}^{n}$ defined by

$$
L(u)=\left(\Delta u_{0}, \Delta u_{1}, \ldots, \Delta u_{n-2}, u_{0}-u_{n-1}\right)
$$

is such that

$$
\operatorname{Ker}(L)=\{(c, \ldots, c): c \in \mathbb{R}\}, \quad \operatorname{Im}(L)=\left\{y \in \mathbb{R}^{n}: \sum_{m=0}^{n-1} y_{m}=0\right\}
$$


so that Assumption (3) holds, with $\bar{u}:=\frac{1}{n} \sum_{m=0}^{n-1} u_{m}$, and Assumption (4) is a direct consequence of the finite dimension of $\widetilde{\mathcal{C}}$ and $\widetilde{\mathcal{L}}$. Thus, all conditions of Theorem 1 hold for (13).

To obtain more information about the corresponding $\lambda_{-}$and $\lambda_{+}$, notice that problem (13) is equivalent to the linear algebraic system in $\mathbb{R}^{n}$

$u_{m+1}+(\lambda-1) u_{m}=f_{m} \quad(0 \leq m \leq n-2), \quad u_{0}+(\lambda-1) u_{n-1}=f_{n-1}$.

Such a system is uniquely solvable if and only if $(1-\lambda)^{n} \neq 1$, and a direct step-by-step computation gives, for those $\lambda$ the solution

$$
\begin{array}{r}
u_{m}=\frac{1}{1-(1-\lambda)^{n}}\left[\sum_{k=0}^{m-1}(1-\lambda)^{m-k-1} f_{k}+\sum_{k=m}^{n-1}(1-\lambda)^{n+m-k-1} f_{k}\right] \\
\quad(0 \leq m \leq n-1) .
\end{array}
$$

Consequently, if $f_{m} \geq 0$ for all $0 \leq m \leq n-1$, we will have $u_{m} \geq 0$ for all $0 \leq m \leq n-1$ if $1-(1-\lambda)^{n}$ and all powers of $(1-\lambda)$ have the same sign. The only possible case is when they are both positive, i.e. when $0<\lambda<1$. Similarly, we will have $u_{m} \leq 0$ for all $0 \leq m \leq n-1$ if $1-(1-\lambda)^{n}$ and all powers of $(1-\lambda)$ have opposite signs. The only possible case is where $1-(1-\lambda)^{n} \leq 0$ and all powers of $(1-\lambda)$ are positive, i.e. when $\lambda<0$. Thus for problem (13)

$$
-\infty=\lambda_{-}<0<\lambda_{+}=1 .
$$

So we necessarily have $\Lambda_{-}=-\infty$. Now, the (complex) spectrum of $-L$ is the set of $\lambda \in \mathbb{C}$ such that $(1-\lambda)^{n}=1$, and hence is made of the $n$ eigenvalues

$$
\lambda_{m}=1-e^{\frac{2 \pi m i}{n}} \quad(0 \leq m \leq n-1),
$$

which are located on the circle of center 1 and of radius 1 . This implies that $\Lambda_{+}=1$.

Notice the difference between the periodic problem for difference equations (13) and the corresponding periodic problem for an ordinary differential equation

$$
u^{\prime}+\lambda u=f(t), \quad u(0)=u(2 \pi)
$$

considered in Subsection 3.1. We had found in this case

$$
-\infty=\Lambda_{-}=\lambda_{-}, \quad \lambda_{+}=\Lambda_{+}=+\infty .
$$

The difference is related to the nature of the spectra of the two problem : a purely imaginary one for $(15)$, and a spectrum on the circle $\partial B(1,1)$ for (13). 


\subsection{A less standard two-point boundary value problem}

We now derive a maximum principle for the boundary value problem

$$
\left\{\begin{array}{l}
u^{\prime \prime \prime \prime}+\lambda u=f(t) \\
u^{\prime}(0)=u^{\prime \prime}(0)=u^{\prime}(\pi)=u^{\prime \prime}(\pi)=0
\end{array}\right.
$$

To this end we consider the space $\Omega=[0, \pi]$ with the measure $d \mu=v(t) d t$, where $v(t):=t(\pi-t)$. This choice of $\mu$ leads to the space of integrable functions

$$
\mathcal{L}=\left\{f:[0, T] \rightarrow \mathbb{R} \mid f \text { is measurable and } \int_{0}^{\pi}|f(s)| v(s) d s<\infty\right\}
$$

with the norm

$$
\|f\|_{1}=\int_{0}^{\pi}|f(t)| v(t) d t .
$$

The operator $L u=u^{\prime \prime \prime \prime}$ is defined on the space $\operatorname{Dom}(L)$ composed by those functions $u \in C^{3}[0, \pi]$ such that

$$
u^{\prime}(0)=u^{\prime \prime}(0)=u^{\prime}(\pi)=u^{\prime \prime}(\pi)=0
$$

and the fourth derivative $u^{\prime \prime \prime \prime}$ belongs to $\mathcal{L}$. This derivative is understood in the sense of distributions. It is easy to check that

$$
\operatorname{Ker}(L)=\{\text { constant functions }\}
$$

To compute the range we split the problem

$$
L u=f, \quad u \in \operatorname{Dom}(L), f \in \mathcal{L}
$$

in two second order problems. First we observe that $z=u^{\prime \prime}$ satisfies

$$
\left\{\begin{array}{l}
z^{\prime \prime}=f(t) \\
z(0)=z(\pi)=0
\end{array}\right.
$$

This Dirichlet problem has the unique solution $u(t)=\int_{0}^{\pi} G(t, s) f(s) d s$ with

$$
G(t, s)= \begin{cases}\frac{(s-\pi) t}{\pi} & 0<t \leq s \\ \frac{(t-\pi) s}{\pi} & s<t<\pi\end{cases}
$$

Notice that $|G(t, s)| \leq \frac{v(s)}{\pi}$ and so the integral makes sense if $f \in \mathcal{L}$. 
Next we must solve the Neumann problem

$$
\left\{\begin{array}{l}
u^{\prime \prime}=z(t) \\
u^{\prime}(0)=u^{\prime}(\pi)=0
\end{array}\right.
$$

This is solvable if and only if

$$
0=\int_{0}^{\pi} z(t) d t=\int_{0}^{\pi} f(s) \int_{0}^{\pi} G(t, s) d t d s=-\frac{1}{2} \int_{0}^{\pi} f(s) v(s) d s .
$$

From here we deduce that $\operatorname{Im}(L)$ is precisely $\widetilde{\mathcal{L}}$. When $f \in \widetilde{\mathcal{L}}$ the Neumann problem has a continuum of solutions but only one of them lies in $\widetilde{\mathcal{C}}$. This fact leads to the unique solvability of

$$
L \widetilde{u}=\widetilde{f}, \quad \widetilde{u} \in \widetilde{\mathcal{C}}, \quad \widetilde{f} \in \widetilde{\mathcal{L}} .
$$

The above discussions imply in particular that $\|z\|_{\infty} \leq \frac{1}{\pi}\|f\|_{1}$ and from here it is easy to arrive at the estimate $\|\widetilde{u}\|_{\infty} \leq K\|\widetilde{f}\|_{1}$. We are in the conditions of Theorem 1 and so we have obtained a maximum principle for (16).

\subsection{The polyharmonic operator with Neumann boundary conditions}

The classical maximum principles for the Laplace operator cannot be proved using our abstract setting. To illustrate the reasons for this obstruction we consider the Neumann problem

$$
\begin{cases}\Delta u+\lambda u=f(x), & x \in D \\ \frac{\partial u}{\partial n}=0 & \text { on } \partial D\end{cases}
$$

where $D \subset \mathbb{R}^{N}, N \geq 2$, is a bounded domain of class $C^{2}$. From [29], the maximum principle holds for $\lambda<0$ but, as shown in [15], it is not valid for any $\lambda>0$ (no UAMP). That is, $\lambda_{-}=-\infty, \lambda_{+}=0$, a situation which cannot occur when Theorem 1 is applicable. Indeed, if we would try to apply our abstract setting we would be stopped after observing that there is no $L^{1}-L^{\infty}$ regularity for the problem

$$
\begin{cases}\Delta u=\tilde{f}(x), & x \in D \\ \frac{\partial u}{\partial n}=0 & \text { on } \partial D .\end{cases}
$$

Given $\tilde{f} \in L^{1}(D)$ with $\int_{D} \tilde{f}=0$, there exists a unique solution with $\int_{D} \tilde{u}=0$. In general this solution will not be continuous but it belongs to $L^{q}(D)$ with 
$q<\frac{N}{N-2}$. This can be proved using the duality method as employed in the final remarks of Chapter IX in [6]. This method also leads to the estimate $\|\tilde{u}\|_{q} \leq K\|\tilde{f}\|_{1}$. By a solution of (18) we understand a function $u \in L^{q}(D)$ such that

$$
\int_{D} u \Delta \phi=\int_{D} \tilde{f} \phi
$$

for each $\phi \in C^{\infty}(\bar{D})$ with $\frac{\partial \phi}{\partial \eta}=0$ on $\partial D$.

Assume now that $k$ is an integer with

$$
2 k>N
$$

and consider the problem

$$
\begin{cases}\Delta^{k} u=\tilde{f}(x), & x \in D \\ \frac{\partial u}{\partial n}=\frac{\partial}{\partial n} \Delta u=\ldots=\frac{\partial}{\partial n} \Delta^{k-1} u=0 & \text { on } \partial D\end{cases}
$$

where $\Delta^{k}=\Delta \circ \ldots{ }^{(k)} \circ \Delta$ is the polyharmonic operator. This problem is equivalent to the system

$$
\begin{cases}\Delta v_{i}=v_{i+1}, & \text { in } D, \quad i=0,1, \ldots, k-1 \\ \frac{\partial v_{i}}{\partial n}=0 & \text { on } \partial D, \quad i=0,1, \ldots, k-2 \\ v_{0}=u, \quad v_{k}=\tilde{f} . & \end{cases}
$$

Now it is easy to prove that if $\tilde{f} \in \tilde{L}^{1}(D)$ then there exists a unique solution $\tilde{u} \in \tilde{C}(\bar{D})$ with $\|\tilde{u}\|_{\infty} \leq K\|\tilde{f}\|_{1}$. To prove this we select $q$ satisfying

$$
\frac{N}{N-2}>q, \quad \frac{1}{q}-\frac{2(k-1)}{N}<0 .
$$

The second inequality is imposed to guarantee that a certain Sobolev space is contained in $C(\bar{D})$. From the previous discussion for (18) we know that $v_{k-1} \in L^{q}(D)$ and, by standard elliptic regularity, $u \in W^{2(k-1), q}(D) \subset$ $C(\bar{D})$. This discussion shows that Theorem 1 can be applied to the problem below when $k>2 N$,

$$
\begin{cases}\Delta^{k} u+\lambda u=f(x), & x \in D \\ \frac{\partial u}{\partial n}=\frac{\partial}{\partial n} \Delta u=\ldots=\frac{\partial}{\partial n} \Delta^{k-1} u=0 & \text { on } \partial D\end{cases}
$$

In this case $\Omega=\bar{D}, \mu$ is the Lebesgue measure and $L u=\Delta^{k} u$. The definition of the domain of $L$ is induced by the formulation of the equation as a system and the duality method. Namely, $\operatorname{Dom}(L)$ is composed by those functions $u \in C(\bar{D})$ such that there exist $v_{0}=u, v_{1}, \ldots, v_{k} \in L^{1}(D)$ satisfying

$$
\int_{D} v_{i} \Delta \phi=\int_{D} v_{i+1} \phi, \quad I=0,1, \ldots, k-1
$$


for each $\phi \in C^{\infty}(\bar{D})$ with $\frac{\partial \phi}{\partial \eta}=0$ on $\partial D$. Notice that $L u=u_{k}$.

Maximum or anti-maximum principles for the polyharmonic operator and Dirichlet boundary conditions have been obtained in [13, 14, 19, 20, 22].

\subsection{Some hyperbolic equations on a torus}

The space $\Omega$ is here the two dimensional torus $\mathbb{T}^{2}=(\mathbb{R} / 2 \pi \mathbb{Z})^{2}$ with the associated Haar measure $\mu$. It is assumed that $\mu\left(\mathbb{T}^{2}\right)=(2 \pi)^{2}$ and generic points on the torus are denoted by $(\bar{t}, \bar{x})$ with $\bar{t}=t+2 \pi \mathbb{Z}, \bar{x}=x+2 \pi \mathbb{Z}$.

We consider the differential operator

$$
L u=\partial_{t}^{2} u+c \partial_{t} u+(-1)^{\gamma} \partial_{x}^{2 \gamma} u
$$

acting on doubly periodic functions $u: \mathbb{T}^{2} \rightarrow \mathbb{R}, u=u(t, x), c>0$, and $\gamma=1,2, \ldots$ This operator is related to the model of telegraph transmission for $\gamma=1$ and to the vibration of beams for $\gamma=2$. The derivatives of $u$ are understood as distributions on the torus so that

$$
<L u, \phi>=\int_{\mathbb{T}^{2}} u L^{\star} \phi
$$

for each $\phi \in \mathcal{D}\left(\mathbb{T}^{2}\right)=C^{\infty}\left(\mathbb{T}^{2}\right)$, where $L^{\star}=\partial_{t}^{2}-c \partial_{t} u+(-1)^{\gamma} \partial_{x}^{2 \gamma}$ is the adjoint operator. We consider $L$ as an operator from $\mathcal{C}=C\left(\mathbb{T}^{2}\right)$ to $\mathcal{L}=L^{1}\left(\mathbb{T}^{2}\right)$ with

$$
\operatorname{Dom}(L)=\left\{u \in C\left(\mathbb{T}^{2}\right) \mid L u \in L^{1}\left(\mathbb{T}^{2}\right)\right\} .
$$

We claim that all the conditions of the abstract setting hold and so we obtain maximum principles for the doubly periodic solutions of

$$
\partial_{t}^{2} u+c \partial_{t} u+(-1)^{\gamma} \partial_{x}^{2 \gamma} u+\lambda u=f(t, x) \text { in } \mathcal{D}^{\prime}\left(\mathbb{T}^{2}\right)
$$

whenever $\lambda \in\left[\lambda_{-}, \lambda_{+}\right] \backslash\{0\}$. For $\gamma=1$ this is the maximum principle found in [27]. The result seems to be new for $\gamma=1$ and $\lambda \in\left[\lambda_{-}, 0\right)$ or for $\gamma \geq 2$.

To justify that $L$ satisfies the conditions of the abstract setting we will assume that $\gamma \geq 2$. The case $\gamma=1$ follows from Proposition 4.4 in [27]. First we state an auxiliary result for equation

$$
\partial_{t}^{2} u+c \partial_{t} u+(-1)^{\gamma} \partial_{x}^{2 \gamma} u+\frac{c^{2}}{4} u=f(t, x) \text { in } \mathcal{D}^{\prime}\left(T^{2}\right)
$$

Lemma 4 For each $f \in L^{1}\left(\mathbb{T}^{2}\right)$ equation (19) has a unique solution $u$ in $C\left(\mathbb{T}^{2}\right)$. Moreover the resolvent operator

$$
R: L^{1}\left(\mathbb{T}^{2}\right) \rightarrow C\left(\mathbb{T}^{2}\right), f \rightarrow u
$$

is compact if $\gamma \geq 2$. 
Notice that in the abstract setting the map $R$ corresponds to the resolvent $R_{\frac{c^{2}}{4}}$.

${ }^{4}$ We postpone the proof of this lemma, and first discuss how to apply it. By selecting the test function $\phi(t, x)=e^{i(n t+m x)}$ it is easy to conclude that

$$
\operatorname{Ker}(L)=\{\text { constant functions }\}
$$

The choice $\phi \equiv 1$ lead to

$$
\operatorname{Im}(L) \subset \widetilde{\mathcal{L}}
$$

It remains to prove that this inclusion is indeed an equality and that the generalized inverse is continuous as a map from $\mathcal{L}$ to $\mathcal{C}$. To this end we observe that the equation $L u=f$ can be rewritten as $L u+\frac{c^{2}}{4} u=f+\frac{c^{2}}{4} u$ and, after an application of Lemma 4 we obtain the equivalence

$$
L u=f \Longleftrightarrow\left(I-\frac{c^{2}}{4} R\right) u=R f .
$$

With the symbol $R$ we also indicate the restriction of $R$ to $\mathcal{C}$. This restriction becomes a compact endomorphism of $\mathcal{C}$. The previous discussion implies that

$$
\operatorname{Ker}\left(I-\frac{c^{2}}{4} R\right)=\{\text { constant functions }\}, \operatorname{Im}\left(I-\frac{c^{2}}{4} R\right) \subset \widetilde{\mathcal{C}}
$$

To obtain the last inclusion we observe that $R(\widetilde{\mathcal{L}}) \subset \widetilde{\mathcal{C}}$. Since $R$ is compact we notice that $I-\frac{c^{2}}{4} R$ is a Fredholm operator of zero index. This implies that $\operatorname{Im}\left(I-\frac{c^{2}}{4} R\right)=\widetilde{C}$ and so $\operatorname{Im}(L)=\widetilde{\mathcal{C}}$. The continuity of the generalized inverse can be deduced as a consequence of the general theory of compact operators.

It is possible to obtain some upper estimates of the numbers $\left|\lambda_{-}\right|$and $\lambda_{+}$. The spectrum of $L$ is given by

$$
\sigma(L)=\left\{n^{2}-m^{2 \gamma}+\operatorname{cin} \mid(n, m) \in \mathbb{Z} \times \mathbb{Z}\right\}
$$

implying that $-\frac{1}{2} \leq \lambda_{-}<0<\lambda_{+} \leq \frac{c^{2}+1}{2}$.

We conclude with the

Proof of Lemma 4. The uniqueness follows from Fourier analysis. Again one uses $e^{i(n t+m x)}$ as test functions. To prove the existence of a solution and the 
compactness of the resolvent we construct a fundamental solution of $L+\frac{c^{2}}{4} I$ on the torus. This is a function $U \in C\left(\mathbb{T}^{2}\right)$ satisfying

$$
L U+\frac{c^{2}}{4} U=\delta \text { in } \mathcal{D}^{\prime}\left(\mathbb{T}^{2}\right)
$$

Here $\delta \in D^{\prime}\left(\mathbb{T}^{2}\right)$ is the Dirac measure concentrated at $(\overline{0}, \overline{0})$ that is

$$
<\delta, \phi>=\phi(0,0) \text { for each } \phi \in \mathcal{D}\left(\mathbb{T}^{2}\right) .
$$

Once the function $U$ has been constructed the solution of $\left(L+\frac{c^{2}}{4} I\right) u=f$ can be expressed as a convolution on $\mathbb{T}^{2}$, namely

$$
u(t, x)=(U * f)(t, x)=\int_{\mathbb{T}^{2}} U(t-\tau, x-\xi) f(\tau, \xi) d \tau d \xi
$$

The compactness of $\mathcal{R} f=U * f$ is now a consequence of Ascoli-Arzelá's theorem.

After this discussion it remains to construct $U$. It is defined as the uniformly convergent series $U=\sum_{n=0}^{\infty} U_{n}$, where $U_{n} \in C\left(\mathbb{T}^{2}\right) \cap C^{2}([0, \pi] \times \mathbb{R})$ satisfies

$$
L U_{n}+\frac{c^{2}}{4} U_{n}=0 \text { on }[0,2 \pi] \times \mathbb{R}
$$

and

$$
\frac{\partial U_{n}}{\partial t}\left(0^{+}, x\right)-\frac{\partial U_{n}}{\partial t}\left(2 \pi^{-}, x\right)= \begin{cases}\frac{1}{2 \pi} & \text { if } n=0 \\ \frac{1}{\pi} \cos n x & \text { if } n>1 .\end{cases}
$$

Assuming by now that the function $U_{n}$ has been constructed we explain why $U$ is the fundamental solution. Integration by parts leads to

$$
\int_{\mathbb{T}^{2}}\left(L^{*} \phi+\frac{c^{2}}{4} \phi\right) U_{n}=\int_{0}^{2 \pi}\left(\frac{\partial U_{n}}{\partial t}\left(0^{+}, x\right)-\frac{\partial U_{n}}{\partial t}\left(2 \pi^{-}, x\right)\right) \phi(0, x) d x
$$

and so

$$
\begin{aligned}
\int_{\mathbb{T}^{2}}\left(L^{*} \phi+\frac{c^{2}}{4} \phi\right) U & =\frac{1}{2 \pi} \int_{0}^{2 \pi} \phi(0, x) d x+\frac{1}{\pi} \sum_{n=1}^{\infty} \int_{0}^{2 \pi} \phi(0, x) \cos n x d x \\
& =\phi(0,0)
\end{aligned}
$$

The last identity follows from the Fourier expansion of $\phi(0, \cdot)$.

Finally we give an explicit formula for $U_{n}$. For $n \geq 1, U_{n}$ is the periodic extension of

$$
U_{n}(t, x)=\frac{e^{-c t / 2}}{\pi\left(1-e^{-c \pi}\right)} \frac{\sin n^{\gamma} t \cos n x}{n^{\gamma}},(t, x) \in[0,2 \pi] \times \mathbb{R} .
$$


For $n=0$ the function $U_{0}$ does not depends on $x$ and is defined as the periodic extension of solution of the boundary value problem

$$
\left\{\begin{array}{l}
u^{\prime \prime}+c u^{\prime}+\frac{c^{2}}{4} u=0, t \in[0,2 \pi] \\
u(0)=u(2 \pi), u^{\prime}\left(0^{+}\right)=u^{\prime}\left(2 \pi^{-}\right)+\frac{1}{2 \pi} .
\end{array}\right.
$$

Namely,

$$
U_{0}(t)=\left[\frac{e^{-c \pi}}{\left(1-e^{-c \pi}\right)^{2}}+\frac{t}{2 \pi\left(1-e^{-c \pi}\right)}\right] e^{-c t / 2}, t \in[0,2 \pi] .
$$

\section{References}

[1] B. Alziary, J. Fleckinger, P. Takáč, An extension of maximum and antimaximum principles to a Schrödinger equation in $\mathbb{R}^{2}$, J. Differential Equations 156 (1999), 122-152

[2] D. Arcoya, J.L.Gámez, Bifurcation theory and related problems : antimaximum principle and resonance, Comm. Partial Differential Equations 26 (2001), 1879-1911

[3] I.V. Barteneva, A. Cabada, A.O. Ignatyev, Maximum and antimaximum principles for the general operator of second order with variable coefficients, Appl. Math. Comput. 134 (2003), 173-184

[4] H. Berestycki, L. Nirenberg, S.R.S Varadhan, The principal eigenvalue and maximum principle for second order elliptic operators in general domains, Comm. Pure Appl. Math. 47 (1994), 47-92

[5] I. Birindelli, Hopf's lemma and anti-maximum principle in general domains, J. Differential Equations 119 (1995), 450-472

[6] H. Brezis, Analyse fonctionnelle, Masson, Paris, 1983

[7] A. Cabada, The method of lower and upper solutions for second, third, fourth and higher order boundary value problems, J. Math. Anal. Appl. 185 (1994), 302-320

[8] A. Cabada, The method of lower and upper solutions for $\mathrm{n}^{\text {th }}$ order periodic boundary value problems, J. Appl. Math. Stochastic Anal. 7 (1994), 33-47 
[9] A. Cabada, The method of lower and upper solutions for third order periodic boundary value problems, J. Math. Anal. Appl. 195 (1995), $568-589$

[10] A. Cabada, S. Lois, Maximum principles for fourth and sixth order periodic boundary value problems, Nonlinear Anal. 29 (1997), 11611171

[11] A. Cabada, L. Sanchez, A positive operator approach to the Neumann problem for a second order ordinary differential equation, J. Math. Anal. Appl. 204 (1996), 774-785

[12] Ph. Clément, L.A. Peletier, An anti-maximum principle for secondorder elliptic operators, J. Differential Equations 34 (1979), 218-229

[13] Ph. Clément, G. Sweers, Uniform anti-maximum principles, J. Differential Equations 164 (2000), 118-154

[14] Ph. Clément, G. Sweers, Uniform anti-maximum principle for polyharmonic boundary value problems, Proc. Amer. Math. Soc. 129 (2000) $467-474$

[15] D.G. De Figueiredo, J.P. Gossez, On the first curve of the Fučik spectrum of an elliptic operator, Differential Integral Equations 7 (1994), $1285-1302$

[16] J. Dieudonné, Éléments d'analyse 2, $2^{e}$ éd., Gauthier-Villars, Paris, 1974

[17] T. Godoy, J.P. Gossez, S. Paczka, Antimaximum principle for elliptic problems with weight, Electronic J. Differential Equations 1999 (1999), No. 22, 1-15

[18] T. Godoy, J.P. Gossez, S. Paczka, A minimax formula for principal eigenvalues and application to an antimaximum principle, Calculus of Variations 21 (2004), 85-111

[19] H.C. Grunau, G. Sweers, Positivity for equations involving polyharmonic operators with Dirichlet boundary conditions, Math. Ann. 307 (1997), 589-626

[20] H.C. Grunau, G. Sweers, Optimal conditions for anti-maximum principles, Ann. Scuola Norm. Sup. Pisa Cl. Sci. (4) 30 (2001), 499-513 
[21] P. Hess, An antimaximum principle for linear elliptic equations with an indefinite weight function, J. Differential Equations 41 (1981), 369-374

[22] E. Massa, Superlinear equations and an uniform anti-maximum principle for the multi-laplacian operator, Electronic J. Differential Equations 2004 (2004), No. 97, 1-19

[23] J. Mawhin, R. Ortega, A.M. Robles-Pérez, A maximum principle for bounded solutions of the telegraph equations and applications to nonlinear forcings, J. Math. Anal. Appl. 251 (2000), 695-709.

[24] J. Mawhin, R. Ortega, A.M. Robles-Pérez, Maximum principles for bounded solutions of the telegraph equation in space dimensions two and three and applications, J. Differential Equations 208 (2005), 42-63

[25] J.J. Nieto, Periodic solutions for third order ordinary differential equations, Comment. Math. Univ. Carolin. 32 (1991), 495-499

[26] P. Omari, M. Trombetta, Remarks on the lower and upper solutions method for second and third-order periodic boundary value problems, Appl. Math. Comput. 50 (1992), 1-21

[27] R. Ortega, A.M. Robles-Pérez, A maximum principle for periodic solutions of the telegraph equation, J. Math. Anal. Appl. 221 (1998), $625-651$

[28] Y. Pinchover, Maximum and anti-maximum principles and eigenfunctions estimates via perturbation theory of positive solutions of elliptic equations, Math. Ann. 314 (1999), 555-590

[29] M. H. Protter, H. Weinberger, Maximum Principles in Differential Equations, Prentice Hall, Englewoods Cliffs, N.J., 1967

[30] P. Pucci, J. Serrin, The Maximum Principle, Birkhäuser, Basel, 2007

[31] W. Reichel, Sharp parameter ranges in the uniform anti-maximum principle for secnd-order ordinary differential operators, Z. angew. Math. Phys. 54 (2003), 822-838

[32] Junping Shi, A new proof of anti-maximum principle via a bifurcation approach, Results Math. 48 (2005), 162-167

[33] N. Stavrakakis, F. de Thélin, Principal eigenvalues and antimaximum principle for some quasilinear elliptic equation on $\mathbb{R}^{N}$, Math. Nachr. 212 (2000), 155-171 
[34] G. Sweers, $L^{n}$ is sharp for the Anti-maximum principle, J. Differential Equations 134 (1997), 148-153

[35] P. Takáč, An abstract form of maximum and anti-maximum principles of Hopf's type, J. Math. Anal. Appl. 201 (1996) 339-364 